\title{
Aggregating Heterogeneous Sensor Ontologies with Fuzzy Debate Mechanism
}

\author{
Xingsi Xue $\mathbb{D}^{1},{ }^{1}$ Xiaojing Wu $\mathbb{D}^{1},{ }^{1}$ Jie Zhang, ${ }^{2}$ Lingyu Zhang $\mathbb{D}^{3}{ }^{3}$ Hai Zhu $\mathbb{D},{ }^{4}$ \\ and Guojun Mao ${ }^{1}$ \\ ${ }^{1}$ Fujian Provincial Key Laboratory of Big Data Mining and Applications, Fujian University of Technology, Fuzhou, \\ Fujian, 350118, China \\ ${ }^{2}$ School of Computer Science and Engineering, Yulin Normal University, Yulin, Guanxi, 537000, China \\ ${ }^{3}$ School of Computer Science and Mathematics, Fujian University of Technology, Fuzhou, Fujian, 350118, China \\ ${ }^{4}$ School of Network Engineering, Zhoukou Normal University, Zhoukou, Henan, 466001, China \\ Correspondence should be addressed to Xingsi Xue; jack8375@gmail.com
}

Received 5 April 2021; Revised 29 April 2021; Accepted 15 May 2021; Published 27 May 2021

Academic Editor: James Ying

Copyright (c) 2021 Xingsi Xue et al. This is an open access article distributed under the Creative Commons Attribution License, which permits unrestricted use, distribution, and reproduction in any medium, provided the original work is properly cited.

\begin{abstract}
Aiming at enhancing the communication and information security between the next generation of Industrial Internet of Things (Nx-IIoT) sensor networks, it is critical to aggregate heterogeneous sensor data in the sensor ontologies by establishing semantic connections in diverse sensor ontologies. Sensor ontology matching technology is devoted to determining heterogeneous sensor concept pairs in two distinct sensor ontologies, which is an effective method of addressing the heterogeneity problem. The existing matching techniques neglect the relationships among different entity mapping, which makes them unable to make sure of the alignment's high quality. To get rid of this shortcoming, in this work, a sensor ontology extraction method technology using Fuzzy Debate Mechanism (FDM) is proposed to aggregate the heterogeneous sensor data, which determines the final sensor concept correspondences by carrying out a debating process among different matchers. More than ever, a fuzzy similarity metric is presented to effectively measure two entities' similarity values by membership function. It first uses the fuzzy membership function to model two entities' similarity in vector space and then calculate their semantic distance with the cosine function. The testing cases from Bibliographic data which is furnished by the Ontology Alignment Evaluation Initiative (OAEI) and six sensor ontology matching tasks are used to evaluate the performance of our scheme in the experiment. The robustness and effectiveness of the proposed method are proved by comparing it with the advanced ontology matching techniques.
\end{abstract}

\section{Introduction}

In the research era of the Next generation of Industrial Internet of Things (Nx-IIoT), the network technology and intelligent computing has become a huge technical model for the government to establish a smart world $[1,2]$. Security issues in the Internet of Things (IoT) have also sparked concern with researches rolling in. Particularly, Xiong et al. [3] proposed a LightPrivacy scheme to achieve the tradeoff between user's personalization privacy protection and the availability of task data in mobile group awareness, whose computational efficiency was significantly improved. Later, they further presented an ATG framework, which was both effective and efficient, and suitable for IoT Mobile Edge Crowd Sensing (MECS) [4]. More recently, Lin et al. [5] proposed an Ant Colony Optimization (ACO) approach to protect information by the transaction deletion, which was able to reduce the side effects while keeping the overall computing cost low. In this fashion, a large number of physical objects embedded with sensors devices exchange information through heterogeneous networks in various applications such as the smart grid, electronic medical 
treatment, and smart cities [6-10]. To aggregate the information of systems efficiently, their entities should be able to interact with one another in meaningful ways without special effort by humans or machines. And it is worth mentioning that many diverse sensor data management application frameworks have been proposed for uniting and dealing sensors. At the same time, value-added information is provided by spatial data sources for public applications, including sensor networks such as Global Sensor Network (https://gsn.sourceforge.net/), Hourglass (https://www.eecs. harvard.edu/ syrah/hourglass/), and IrisNet (https://www. intel-iris.net/). However, the heterogeneity of different sensor networks resulting in a lack of interoperability. Therefore, to build a secure Nx-IIoT, the way of aggregate heterogeneous sensor data in different sensor networks is prominent.

Specifically, there are two aspects of work to be done; one is to improve the expressive ability of sensor network models, and the other is to enhance the interaction between sensor networks to achieve data integration. To address the first aspect, an increasing number of sensor ontologies have appeared because of the preponderance of sensor ontology technique, which is able to model the corresponding networks integrally. And to address the other aspect, the ontology matching technique has been on the stage of history in recent years. Furthermore, one of the cutting-edge research institutions in this field is Ontology Alignment Evaluation Initiative (OAEI) (https://oaei. ontologymatching.org/). Recently, ontology alignment extraction technique has been used to strengthen the team. To advance the relevant work, we propose a mechanism for sensor ontology matching with the Fuzzy Debate Mechanism (FDM) based ontology alignment extraction technique, which aims to extract the correct sensor ontology matching pairs in different alignments generated by different basic matching measures. To be specific, we first express the similarity between two sensor entities in the three-dimensional vector space through a fuzzy membership function and then evaluate the similarities in multiple dimensions and the cosine theorem is introduced to evaluate the distance of similarity vectors and the golden one.

The following sections are arranged as follows. Section 2 is an overview of the related work. Section 3 presents a preliminary analysis of the relevant concepts. Details of FDM are provided by Section 4 . Section 5 externalizes experiments' results and makes the corresponding analysis, and Section 6 concludes the work.

\section{Related Work}

A growing number of sensor ontologies have appeared due to the sensor ontology possesses powerful sensor network model expression ability, i.e., SensorOntology 2009 ontology, SSN ontology and IoT-Lite ontology, and so on [11-13]. And to enhance the interaction between sensor networks to achieve data integration, the sensor ontology matching technique has been brought out these years [14].

In the research upsurge, there are two technical routes: ontology meta-matching (OMM) techniques and ontology entity matching techniques. The ontology entity matching techniques try to determine the entity correspondence set between two ontologies directly, while the OMM techniques try to solve the problem of aggregate different similarity measures with appropriate weights [15]. There are plenty of popular technical approaches in computing intelligence (CI) to solve OMM problems, e.g., machine learning (ML), evolutionary computing (EC), and swarm intelligence (SI). For example, many ML technologies [16-21] have been proposed to automatically determine ontology alignment, and experiments have shown that ML greatly improves matching efficiency, and the genetics for ontology alignment (GOAL), which was designed to optimize aggregate weight sets for different matchers [22-25]. To overcome the disadvantage of excessive reliance on reference alignment, Xue et al. put forward the partial reference alignment (PRA) and the unanimous improvement rate (UIR) [26]. Furthermore, Xue et al. proposed a series solution using compact algorithm (CA) and sensor ontology meta-matching technique to aggregate weight sets for different matchers [27].

However, in the existing ontology matching methods based on CI, various ontology matchers are regarded as tools with the same effect and try to aggregate their outputs by determining the optimal weights [28]. The matching quality will decrease if ignoring the influence of different entity mappings on the matching results of different matching devices. Furthermore, adjusting weights with this method can be problematic; that means they may not be reusable in different ontology matching scenarios. Hence, the influence of entity mappings on matching result cannot be ignored, which is addressed by ontology alignment extraction technique [29]. Recently, the context extraction technique has been widely applied in semantic field $[26,30]$, in which OntoLT uses terminology extraction, ontology structure mapping, the statistical method, and the language model of definition to extract ontology concept [31]. Besides, Gaeta et al. implemented several statistical and data mining algorithms to identify and extract the concepts as well as their relationships in ontologies [32]. In addition, the rule of extraction process is typically described as a series of agreement reaching processes, such as argumentation frameworks [33]. In concrete terms, the argumentation framework proposed by Laera et al. relies on preferences between a formal argument operation pattern and a particular type of argument, taking into account ontology-based arguments and propositions specific to the matching task [34]. Dos-Santos and Euzenat used argumentation as a supporting or rejecting parameter and proposed a computational strategy to remove inconsistencies in the result alignment and allow consistency in the argumentation system [35].

It is worth mentioning that the similarity of ontology matching and the relation between entities and the similarity threshold can all be regarded as uncertain problems, in which fuzzy logic is highly adept [36]. In the proposal of [37], the ontology matches are expressed by the fuzzy set of reference concepts or instances that makes the new ontology be directly compared with the original one. Todorov et al. proposed a fuzzy ontology alignment using background 
knowledge [38]. UFOM adopts fuzzy set theory as the general framework of fuzzy ontology matching, which represents many types of correspondences and describe the uncertainty in the process of correspondence discovery [39]. And Cross discusses how to extend the process of ontology concept matching by using similarity measure and integration of fuzzy sets [40].

\section{Problem Definition}

3.1. Sensor Ontologies. An ontology is composed of concept set, attribute set, and instance set, and the ontology $O$ refers to three tuples $\left(C, P_{d}, P_{o}\right)$, among which $C, P_{d}, P_{o}$ refer to concepts set, properties of datatype, and properties of object, respectively, called ontology entities. The semantic sensor network (SSN) (https://purl.oclc.org/NET/ssnx/ssn\#) ontology can be regarded as the authoritative ancestor of sensor ontology [41]. It is an OWL 2 ontology put forward by the W3C Semantic Sensor Network Incubator group (SSN-XG) (https://www.w3.org/2005/Incubator/ssn), which models sensors and observation data and represents sensors in accordance with the function, measurement process, observation data, and so on. SensorOntology 2009 (https://www.w3. org/2005/Incubator/ssn/wiki/SensorOntology2009) ontology developed by Michael Compton, i.e., from CSIRO (Australia) has come into use as the source of the SSN Ontology. And OSSN (https://www.w3.org/ns/ssn) is an ontology established by SSN-XG in the year from 2009 to 2011 [42]. Furthermore, the resources, entities, and services in the Internet of Things (IoT) are summarized by the IoT-Lite (https://www.w3.org/ Submission/2015/SUBM-iot-lite-20151126/) ontology outlines, which is a lightweight ontology and a case of an SSN ontology and the latest version was submitted in 2015 [43]. The Sensors, Observations, Samples, and Actuators (SOSA) (https://www.w3.org/ns/sosa) ontology is designed for a broad target audience and applications that have access to the ontology and was released in 2017. In addition, SOSA acts as a minimal interoperability fallback layer; that is, it defines those public classes and attributes whose data can be securely exchanged between the SSN, its modules, and all information used by SOSA.

\subsection{Ontology Matching and Sensor Ontology Alignment} Extraction. Ontology matching is the process of determining the entity correspondence between source ontology and target ontologies to bridge the semantic gap between them. As shown in Figure 1, the input of ontology matching process is a pair of ontologies to-be-matched, and the output is the final alignment. The research on ontology matching mostly involves the calculation and refinement of similarity measure, but the research on extracting the final alignment from similarity measure matrix, which is called ontology alignment extraction technique, is less.

To be specific, sensor ontology alignment extraction technique works by extracting entity correspondences from different matching suggestions generated from different similarity measures for the same sensor ontology matching task to form the final alignment [28]. The framework of ontology alignment extraction is shown in Figure 1, in which the systems to finish the matching work are regarded as ontology matchers. The set of entity correspondences determined by an ontology matcher is called an ontology alignment. In addition, a corresponding $\operatorname{set} A$ is the alignment between two sensor ontologies, where the entity correspondence is referred to a 4-tuple corr $=\left(e, e^{\prime}, n\right.$, relation), $e$ and $e^{\prime}$ are, respectively, two ontologies' entities, and $n \in[0,1]$ is their confidence value, while relation acts as the equivalence relation.

3.3. Fuzzy Similarity Measure. Since some of the wireless sensor network (WSN) domain concepts have not yet been incorporated into a common dictionary, there is no lexical tool to define the linguistic relationships between all concepts for mapping purposes. To conquer the difficulty, our proposal uses a variety of metrics as tools in similarity calculations. Each tool gives different matching suggestions; the matching results between entities become uncertain information. In this case, a unified framework for representing many different modes of inconclusive information is provided by the use of a fuzzy measure to depict inconclusive information [44]. For this reason, we decide to use the fuzzification process to combine them to get more accurate alignment. In other words, the work of fuzzy similarity measurement adopted here is supplemented by two parts; one is the basic similarity measurements, also known as the entity matching measure (EMM), and the other is the membership function of fuzzification.

An EMM is always described as the function to output similar values with information from two entities as input in the interval $[0,1]$, which always plays the role of a basic ontology matcher. EMM can be grouped into three types: first is the string-based type, second is the linguistics-based type, and the third one is called taxonomy-based EMM.

A string-based EMM outputs the edit distance between entities by considering their IDs, tags, comments, and so on. In this work, two well-known EMMs were used to improve the quality of the matching results, namely, the Levenshtein similarity [45] and the Jaro similarity metric [46]. Given two strings, $s_{e 1}$ and $s_{e 2}$, Levenshtein-similarity is defined in the following:

$$
\operatorname{Levenshtein}\left(s_{e 1}, s_{e 2}\right)=\frac{\max \left(0, \min \left(\left|s_{e 1}\right|,\left|s_{e 2}\right|\right)-d\left(s_{e 1}, s_{e 2}\right)\right)}{\min \left(\left|s_{e 1}\right|,\left|s_{e 2}\right|\right)},
$$

where $\left|s_{e 1}\right|$ and $\left|s_{e 2}\right|$ are, respectively, the length of seland $s_{e 2} ; d\left(s_{e 1}, s_{e 2}\right)$ is the number of required operations to transform $s_{e 1}$ to $s_{e 2}$; given the strings $s$ and $t$, define $s^{\prime}$ as a character common to $t$ in $s$; that is, they appear in the same order in $s$ and $t$. Similarly define $t^{\prime}$ in $t$. Now, assume that $s^{\prime}$ and $t^{\prime}$ are transposed at position $i$ when their $i$ th bits are not the same and the value of $T_{s^{\prime}, t^{\prime}}$ is $50 \%$ of the transpositions of $s^{\prime}$ and $t^{\prime}$ in number. According to the assumption above, the Jaro similarity measure of $s$ and $t$ is defined in the following: 


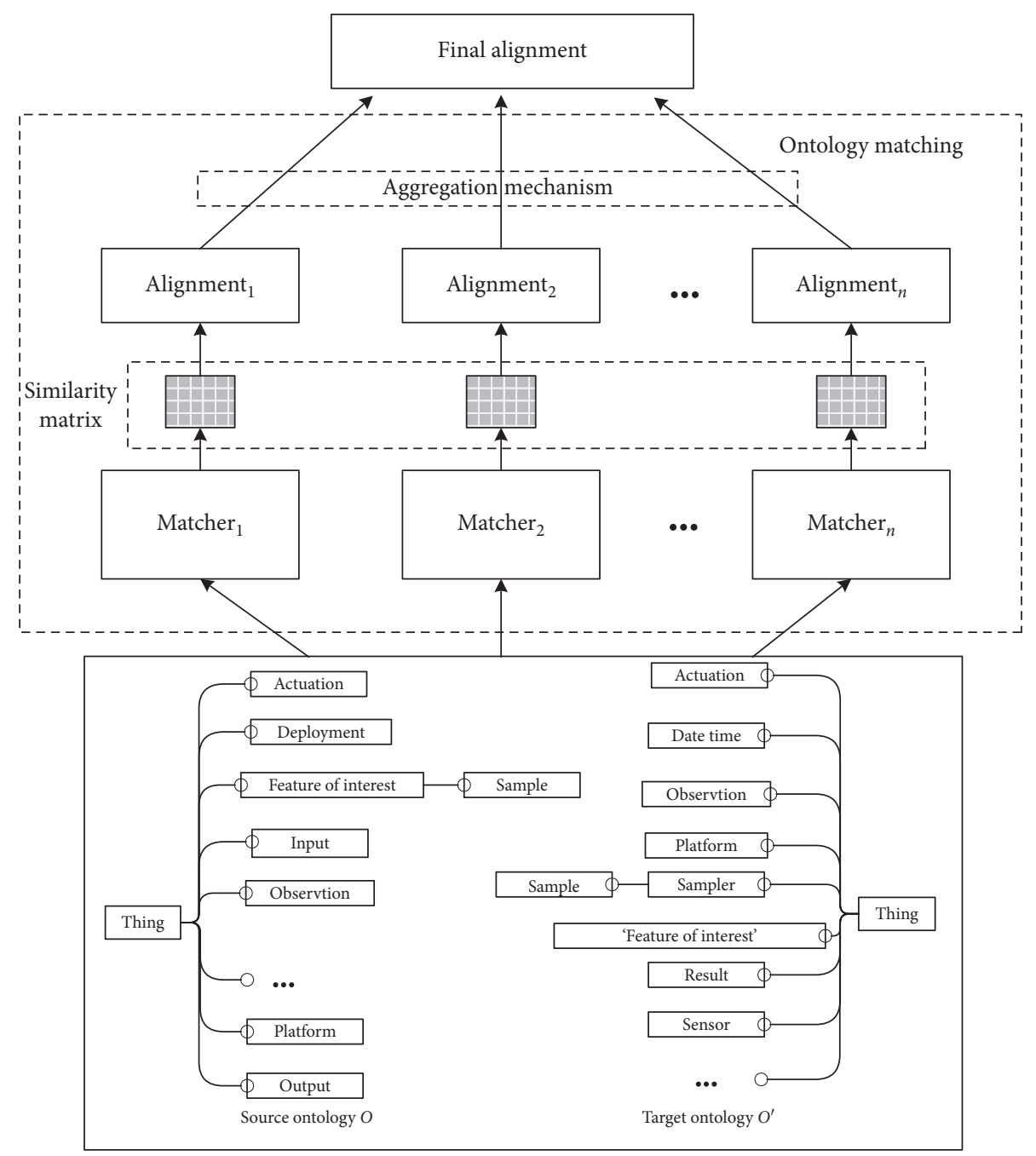

FIgURE 1: Framework of ontology alignment extraction.

$$
\operatorname{Jaro}(s, t)=\frac{1}{3} \cdot\left(\frac{\left|s^{\prime}\right|}{|s|}+\frac{\left|t^{\prime}\right|}{|t|}+\frac{\left|s^{\prime}\right|-T_{s^{\prime}, t^{\prime}}}{\left|s^{\prime}\right|}\right) .
$$

Linguistics-based EMMs often use external corpus or dictionaries (e.g., WordNet [47]) to calculate similarity values through entity tags. Because of the effectiveness of the WordNet, we used WordNet-based distances. Besides, based on the distance WordNet-based on the WordNet electronic vocabulary, the equation is defined as follows:

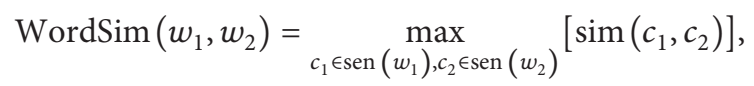

where $w_{1}$ and $w_{2}$ are a word derived from two entities and

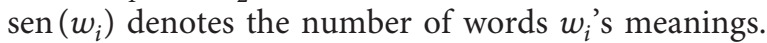

The similarity values obtained by the taxonomy-based EMMs are based on the consideration of the entity structure information as well as the superentity and subentity information. To be specific, in our proposal, the taxonomybased EMM makes use of the structure-based distance calculated by noted algorithm, which adopts a versatile graph matching method called similarity flooding (SF) [48], where an iterative fixpoint computation below is applied to produce congruent relationship between the two ontologies' elements:

$$
\delta^{i+1}=\operatorname{norm}\left(\delta^{i}+f\left(\delta^{i}\right)\right)
$$

In this formula, norm is the normalized process, $\delta^{i}$ is the value of the last iteration that changes in each iteration, and $f$ is a function of increasing the similarity of pairs of elements based on the similarity of their adjacent elements.

The flowchart of the fuzzy similarity measure application process is depicted in Figure 2. The work in pre-processing generally includes conversion ontology format and ontology analysis. And the similarity matrixes are generated from the four basically similar measures adopted in this article. Usually, a matcher determines whether two entities are correct matching pairs by comparing the threshold value with the similarity value. But it is difficult to find an accurate threshold to make the matching result completely correct. Therefore, we introduce the membership function in fuzzy theory and consider the similarity value from the "low," 
"medium," and "high" dimensions. The fuzzy process changes the elements in the similarity matrixes and changes them into vectors through membership function before participating in the Debate Mechanism. In this work, the membership function $\mu(x)$ of fuzzy process is defined as three subfunctions as follows:

$$
\begin{aligned}
& \mu_{\text {Low }}(x)= \begin{cases}-\frac{10}{7} x+1, & x \in[0,0.7), \\
0, & x \in[0.7,1],\end{cases} \\
& \mu_{\text {Medium }}(x)= \begin{cases}2 x, & x \in[0,0.5), \\
-2 x+2, & x \in[0.5,1],\end{cases} \\
& \mu_{\text {High }}(x)=x, \quad x \in[0,1],
\end{aligned}
$$

where $x$ is an element of a similarity matrix which stands for the similarity $n$. According to previous practical experience, we believe that matching pairs with similarity less than 0.7 are mostly mismatched, and 0.5 is a medium similarity in similarity interval $[0,1][28]$. Therefore, $\mu_{\text {Low }}$ is used to measure the degree of low similarity. $\mu_{\text {Medium }}$ describes the degree of medium size and then uses the direct proportionality function to describe the degree of high similarity as the $\mu_{\mathrm{High}}$. After that, we take the three function values as the coordinates of the $3 \mathrm{D}$ vector $\vec{f}$ and input them into the Debate Mechanism.

\section{Debate Mechanism}

In this work, ontology alignment extraction process is carried out by the Debating Mechanism, in which the debating rules is utilised to extract the target information. The Debating Mechanism contains a classification module of correspondences, where a fuzzy measure is built to express the similarities of correspondences, and an argumentation framework, which is used to negotiate different matching suggestions between matchers to reach agreement. In addition, a fuzzy measure is built to express the similarities of correspondences as is shown in Figure 3 that depicts the framework of Debate Mechanism.

The classification module and argumentation framework are described in the following paragraphs.

In the classification module, assume that a matching task is working on two ontologies, $O$ and $O^{\prime}$, by $k(k \geq 2)$ basic ontology matchers. Extend an entity correspondence in an ontology matcher as an argument ar, which is defined as follows:

$$
\text { ar }=\{c, n, v, h\}
$$

where $c=\left(e, e^{\prime}\right), v(v \in N)$, and $h(h \in\{0,1\})$, respectively, express a correspondence, the artificially preset matcher number, and the measure factor of similarity value.
Assume that reference vector $\vec{m}=(0,0,1)$; change the similarity value $n$ into similarity fuzzy vector $\vec{f}=\left(\mu_{\text {Low }}(n), \mu_{\text {Medium }}(n), \mu_{\text {High }}(n)\right)$ in vector space. Then, we describe $h$ as follows:

$$
h= \begin{cases}1, & \text { if } \cos (\vec{f}, \vec{m}) \geq \delta, \\ 0, & \text { if } \cos (\vec{f}, \vec{m})<\delta,\end{cases}
$$

where $\vec{f}$ is a similarity fuzzy vector and $\delta(\delta \in[0,1])$ is set as the similarity's threshold. Especially, while $h=0$, the matcher rejects $c$; otherwise, it accepts it. Suppose that $c$ is allocated to one of the five groups $C_{i}, i=1, \ldots, 5$, that are, respectively, defined as follows: $k(k \geq 2)$ is the number of matchers, $k_{a}$ is the number of matchers that accepts $c, k_{r}$ is the number of matchers that rejects $c, k_{r}=k-k_{a}$. Next, classify $c$ into groups from $C_{1}$ to $C_{5}$ in category column according to the above situation. For detailed classification, see Table 1 .

Since $c \in C_{1}$ is regarded as a correct correspondence which is accepted by all the matchers, while $c \in C_{5}$ is rejected oppositely, the correspondences in groups $C_{1}$ and $C_{5}$ are straightway judged as right correspondences or the false ones in the process of extraction without participating in the following process. Besides, correspondences of $C_{2}, C_{3}$, and $\mathrm{C}_{4}$ groups are in list of the argumentation process.

In the process of argumentation, two arguments are given as $a=\left\{c_{1}, \overrightarrow{f_{1}}, v_{1}, h_{1}\right\}$ and $b=\left\{c_{2}, \overrightarrow{f_{2}}, v_{2}, h_{2}\right\}$. Four relationships are defined between $b$ and $a$, which are unite, attack, support, and disprove. To be specific, unite is marked as $U(b, a)$, and attack, support, and disprove are expressed as $A(b, a), S(b, a)$, and $D(b, a)$. The details are listed in the following descriptions:

(i) When $c_{1}=c_{2}, v_{1} \neq v_{2}, h_{1}=h_{2}, b$ is united with $a$, which is denoted as $U(b, a)$.

(ii) When $c_{1}=c_{2}, v_{1} \neq v_{2}, h_{1} \neq h_{2}, b$ attacks $a$, which is denoted as $A(b, a)$.

(iii) When $\quad c_{2}=C_{2} \rightarrow$ or $\quad c_{2}=C_{3}, \quad v_{1}=v_{2}$, $\cos \left(\overrightarrow{f_{1}}, \vec{m}\right)>\cos \left(\overrightarrow{f_{2}}, \vec{m}\right), \quad h_{1}=h_{2}=1$, or when $c_{2}=C_{4}$ or $C_{3}, \quad \cos \left(\overrightarrow{f_{1}}, \vec{m}\right)<\cos \left(\overrightarrow{f_{2}}, \vec{m}\right)$, $h_{1}=h_{2}=0, b$ supports $a$, which is represented by $S(b, a)$.

(iv) When $v_{1}=v_{2}, c_{1}=C_{i}, c_{2}=C_{j}, i>j(i, j \in(2,3,4))$, $n_{1}>n_{2}, h_{1}=1, h_{2}=0$, or when $v_{1}=v_{2}, c_{1}=C_{i}, c_{2}$ $=C_{j}, i<j(i, j \in(2,3,4)), \quad \cos \left(\overrightarrow{f_{1}}, \vec{m}\right)<\cos \left(\overrightarrow{f_{2}}\right.$, $\vec{m}), h_{1}=0, h_{2}=1, b$ disproves $a$, that is depicted as $D(b, a)$.

The four relationships between arguments are depicted in Figure 4. There are three matchers, i.e., Matcher ${ }_{a}$, Matcher $_{b}$, and Matcher ${ }_{c}$ including their arguments $a_{i}, b_{i}$, and $c_{i}$. Unite and attack happened between arguments from different matchers, but support and disprove from the same one. 
The arguments set is defined as a 7-tuple: \{ar, strength, $U, A, S, D, M\}$, where $U, A, S, D$ are the relationships mentioned above, $M=\left\{m_{1}, m_{2}, \ldots, m_{n}\right\}$ is defined as the set of matchers that contains $n$ basic ontology matchers, argument ar is related to correspondence $c$, and astrength $n \mathrm{~d}$ acts as strength value of $c$ according to a matcher $m_{i}$, which is defined as follows:

$$
\operatorname{Strength}_{c}^{m_{i}}=\cos \left(\sum_{\operatorname{ar} \in A R}\left\{\overrightarrow{f_{x}} \mid x \in A R \wedge S(x, a r)\right\}, \vec{m}\right)-\cos \left(\sum_{\operatorname{ar} \in A R}\left\{\overrightarrow{f_{x}} \mid x \in A R \wedge D(x, a r)\right\}, \vec{m}\right) .
$$

In this paper, $c$ is an element of corresponding arguments existing in every basic ontology matcher, and we need to calculate its judgment factor $r_{c}\left(r_{c} \in\{0,1\}\right)$ whose value is determined in argumentation process to determine whether it can be extracted into the final alignment. Consequently, an essential challenge is to improve the reliability of an entity mapping's judgment element. To meet that challenge, as is defined in formula (9), each matcher's correctness factor is adopted to evaluate the credibility of the matcher depending on how similar it is to the matching recommendations of other matchers. And the support strength and disprove strength in debating process are utilized to offer the evidence of right mappings. In addition, the $r_{c}$ can be obtained after full assessment of the factors mentioned above. follows:

To be specific, the argumentation process is arranged as

Step 1. Apparently, $r_{c}$ is 1 (or 0 ) when $c$ belongs to $C_{1}$ (or $C_{5}$ ), and the similarity values of corresponding rows and columns of $c$ can be deleted from the similarity matrix.

Step 2. Matcher $m_{i}$ 's correctness factor is calculated in the following:

$$
\sigma_{m_{i}}=\frac{\sum_{m_{i}}\left|\left\{c \mid c \in\left(C_{1}, C_{5}\right)\right\}\right|}{\sum_{m_{i}}|c|} .
$$

Step 3. In every matcher, the debating process is brought forward in accordance with the relationships "support" and "disprove":

(1) In the $C_{2}$ group, most matchers support these correspondences. Therefore, the success of the supporting part in defeating the disproving part is calculated. The above situation is explained as follows. The support strength $S s$ of matcher $m_{1}$ is defined as follows:

$$
S s_{t}^{m_{1}}=\cos \left(\sum_{x \in A R} \overrightarrow{f_{x}}, \vec{m}\right)-\cos \left(\sum_{y \in A R} \overrightarrow{f_{y}}, \vec{m}\right),
$$

where argument $x=\left\{c, n_{x}, v_{x}, h_{x}\right\}$, argument $y=$ $\left\{c, n_{y}, v_{y}, h_{y}\right\}, S(x, t), D(y, t)$ and $v_{x}=v_{y}$. When three matchers $m_{1}, m_{2}$, and $m_{3}$ support $c$ but $m_{4}$ disproves it, it is determined that the mapping can be established between $e$ and $e^{\prime}$ when $S s_{t}^{m_{1}}>S s_{t}^{m_{4}}$, $S s_{t}^{m_{2}}>S s_{t}^{m_{4}}$ and $S s_{t}^{m_{3}}>S s_{t}^{m_{4}}$, and $r_{c}$ is set to 1 . Otherwise, $c$ is transformed into $C_{3}$, performing step 4.

(2) In a similar way, for the $C_{4}$ group of argument, it is necessary to calculate whether the attack of the opposing side against the supporting side is successful. Assuming that the matcher $m_{1}$ 's disprove strength $D s$ is defined as follows:

$D s_{t}^{m_{1}}=\cos \left(\sum_{x \in A R} \overrightarrow{f_{x}}, \vec{m}\right)-\cos \left(\sum_{y \in A R} \overrightarrow{f_{y}}, \vec{m}\right)$,

where argument $x=\left\{c, n_{x}, v_{x}, h_{x}\right\}$, argument $y=$ $\left\{c, n_{y}, v_{y}, h_{y}\right\}, D(x, t), S(y, t)$ and $v_{x}=v_{y}$. In this situation, if the three matchers $m_{1}, m_{2}$, and $m_{3}$ disprove $c$, but $m_{4}$ support, obviously, the mapping cannot be established between $e_{i}$ and $e_{j}^{\prime}$ when $D s_{t}^{m_{1}}>D s_{t}^{m_{4}}, D s_{t}^{m_{2}}>D s_{t}^{m_{4}}$ and $D s_{t}^{m_{3}}>D s_{t}^{m_{4}}$, and $r_{c}=0$. Otherwise, $c$ is converted into $C_{3}$ group.

Step 4. For the arguments in $C_{3}$ group, the number of matchers who took the opposite view was almost even, due to what they can be divided into two party called accept party and reject party. Therefore, the core challenge is to figure out which of the two parties defeat the other successfully. To end this, the power $P_{\text {stren }}$ of each party is calculated, which is defined as follows:

$$
P_{\text {stren }}^{\text {party }}=\delta_{m_{1}} \cdot \text { Strength }_{c}^{m_{1}}+\delta_{m_{2}} \cdot \text { Strength }_{c}^{m_{2}}+\cdots+\delta_{m_{n}} \cdot \operatorname{Strength}_{c}^{m_{n}} \text {, }
$$

where $m_{1}, m_{2}, \ldots m_{n}$ are the members of the party. Assume that for $c$, if $P_{\text {stren }}^{\text {accept }} \geq P_{\text {stren }}^{\text {reject }}, r_{c}=1$. Otherwise, $r_{c}=0$.

Step 5. Select the correspondences with $r_{c}=1$, which are further used to decide the final alignment.

\section{Experiment and Results}

5.1. Alignment Evaluation Metric. The alignment can typically be assessed with two measures, often referred to as recall and precision [49], which are severally ruled in the following: 


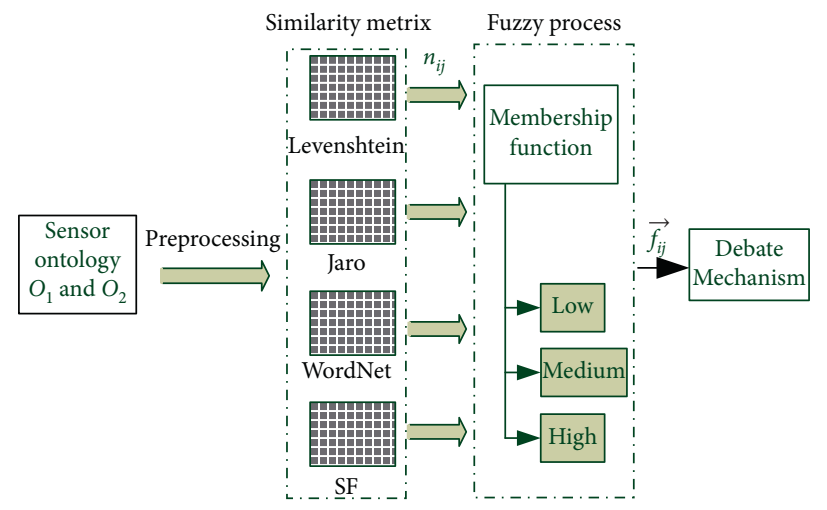

Figure 2: The flowchart of fuzzy similarity measure application process.

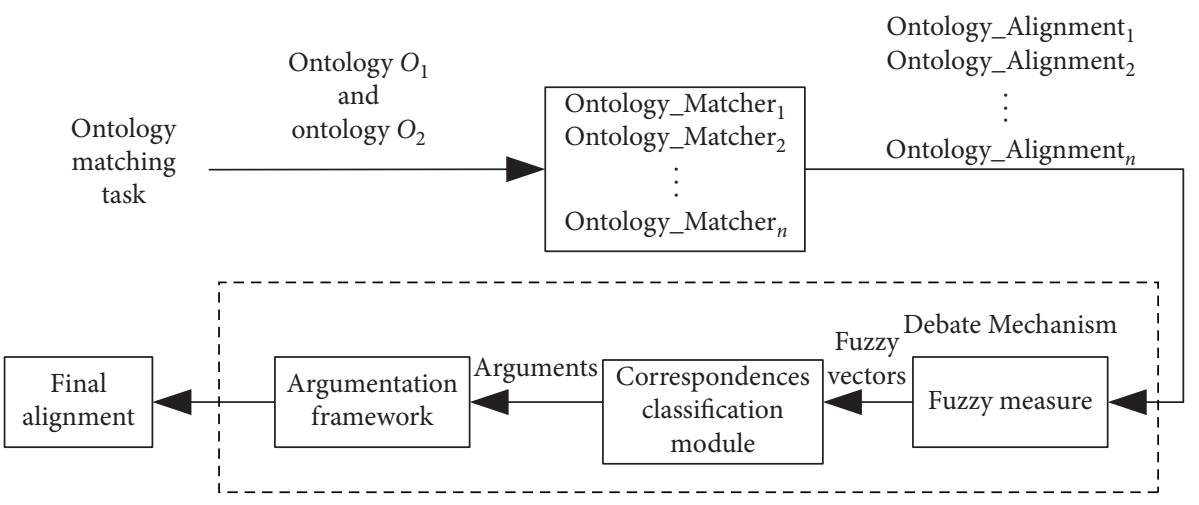

FIgUre 3: The framework of Debate Mechanism.

$$
\begin{aligned}
\text { recall } & =\frac{|R \cap A|}{|R|}, \\
\text { precision } & =\frac{|R \cap A|}{|A|},
\end{aligned}
$$

where the alignment given is $A$, and the reference alignment is $R$. Particularly, recall $=1$ when found all correct matching pairs, recall $=1$. And precision $=1$ stands for that all the matching pairs found are correct. In order to combine the two metrics, the $f$-measure is further employed, which is regarded as a comprehensive measure of recall and precision [38]:

$$
f-\text { measure }=\frac{2 \text { precision } \cdot \text { recall }}{\text { recall }+ \text { precision }} \text {. }
$$

5.2. Experimental Testing Cases. In this experiment, we take advantage of the testing cases in Bibliographic track (https:// oaei.ontologymatching.org/2016/results/benchmarks/index. html) from OAEI as well as six pairs of real sensor ontology matching tasks to verify the sensitivity and availability of our recommendation. Table 2 shows a brief description of OAEI's Bibliographic track, where two ontologies to be mapped and a reference alignment to evaluate the effectiveness of ontology matcher are included by each test case. Table 3 depicts the main features of sensor ontologies.

In this experiment, the similarity threshold, set empirically to 0.85 , guarantees the highest alignment quality achieved on average in all test cases.

5.3. Experimental Results. When performing testing cases from the Bibliographic track, we compare the result of our suggestion with OAEI's participants, i.e., AML, edna, and LogMapLt from the standpoint of $f$-measure. Figure 5 shows the experimental results for all types of testing cases in the Bibliographic track of OAEI.

As can be seen from the presentation information in Figure 5, for most testing cases, our proposal outperformed other methods due to the application of a Debate Mechanism that integrates the advantages of various basic similarity measures; the matching problem can be considered synthetically from different angles in ontology matching. In some cases, the $f$-measure for all matching techniques is nearly zero, which is due to the complexity of testing cases. By contrast, our proposal does a better job in these cases as listed in Table 4, which masks a small step forward in our proposal on the basis of cutting-edge work.

When performing sensor ontology matching tasks, we compare the result of our proposal with four basic EMM that 
TABLE 1: Classification method of correspondences.

\begin{tabular}{lr}
\hline$k_{a}$ & Category \\
\hline$k$ & $C_{1}$ \\
$((k / 2), k)$ & $C_{2}$ \\
$(k / 2)$ & $C_{3}$ \\
$(0,(k / 2))$ & $C_{4}$ \\
0 & $C_{5}$ \\
\hline
\end{tabular}

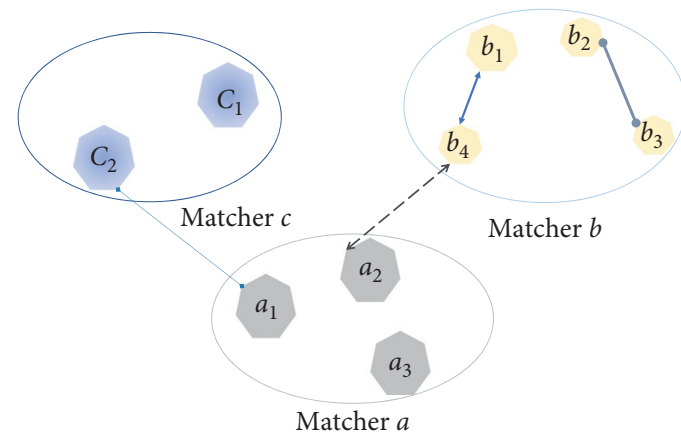

$$
\begin{array}{ll}
\longleftrightarrow- & \text { Unit: } U\left(\mathrm{a}_{2}, \mathrm{~b}_{4}\right) \\
\longleftrightarrow & \text { Attack: } A\left(\mathrm{a}_{1}, \mathrm{c}_{2}\right) \\
\longleftrightarrow & \text { Support: } S\left(\mathrm{~b}_{1}, \mathrm{~b}_{4}\right) \\
\longleftrightarrow & \text { Disprove: } D\left(\mathrm{~b}_{2}, \mathrm{~b}_{3}\right)
\end{array}
$$

FIgURE 4: Four relationships between arguments.

TABle 2: Descriptions on OAEI's Bibliographic track.

\begin{tabular}{lc}
\hline Testing case & Description \\
\hline 101 & Two identical ontologies \\
$201-202$ & Ontologies varying in terminology and semantics characteristics \\
$221-247$ & Ontologies varying in structure characteristics \\
$248-262$ & Ontologies varying in terminology, semantics, and structure characteristics \\
\hline
\end{tabular}

TABle 3: Descriptions on sensor ontologies.

\begin{tabular}{lcr}
\hline Sensor ontology & $\begin{array}{c}\text { Ontology } \\
\text { scale }\end{array}$ & Description \\
\hline $\begin{array}{l}\text { Semantic sensor network ontology (SSN) } \\
\begin{array}{l}\text { Sensor, observation, sample, and actuator } \\
\text { ontology (SOSA) }\end{array}\end{array}$ & $\begin{array}{l}52 \text { entities } \\
\text { IoT-lite ontology (IoT) }\end{array}$ & $\begin{array}{c}\text { It is about sensors, actuators and observations, and related concepts } \\
\text { It defines those common classes and attributes whose data can be securely } \\
\text { exchanged in SSN, its modules, and all SOSA uses }\end{array}$ \\
$\begin{array}{l}\text { SensorOntology2009 ontology (SN) } \\
\begin{array}{l}\text { Original semantic sensor network ontology } \\
\text { (OSSN) }\end{array}\end{array}$ & $\begin{array}{c}\text { It is about key concepts of IoT } \\
107 \text { entities }\end{array}$ & $\begin{array}{c}\text { It is the initial version of the SSN ontology, which was developed in 2009 } \\
\text { It is an original version of SSN, which was developed in 2009-2011 }\end{array}$ \\
\hline
\end{tabular}




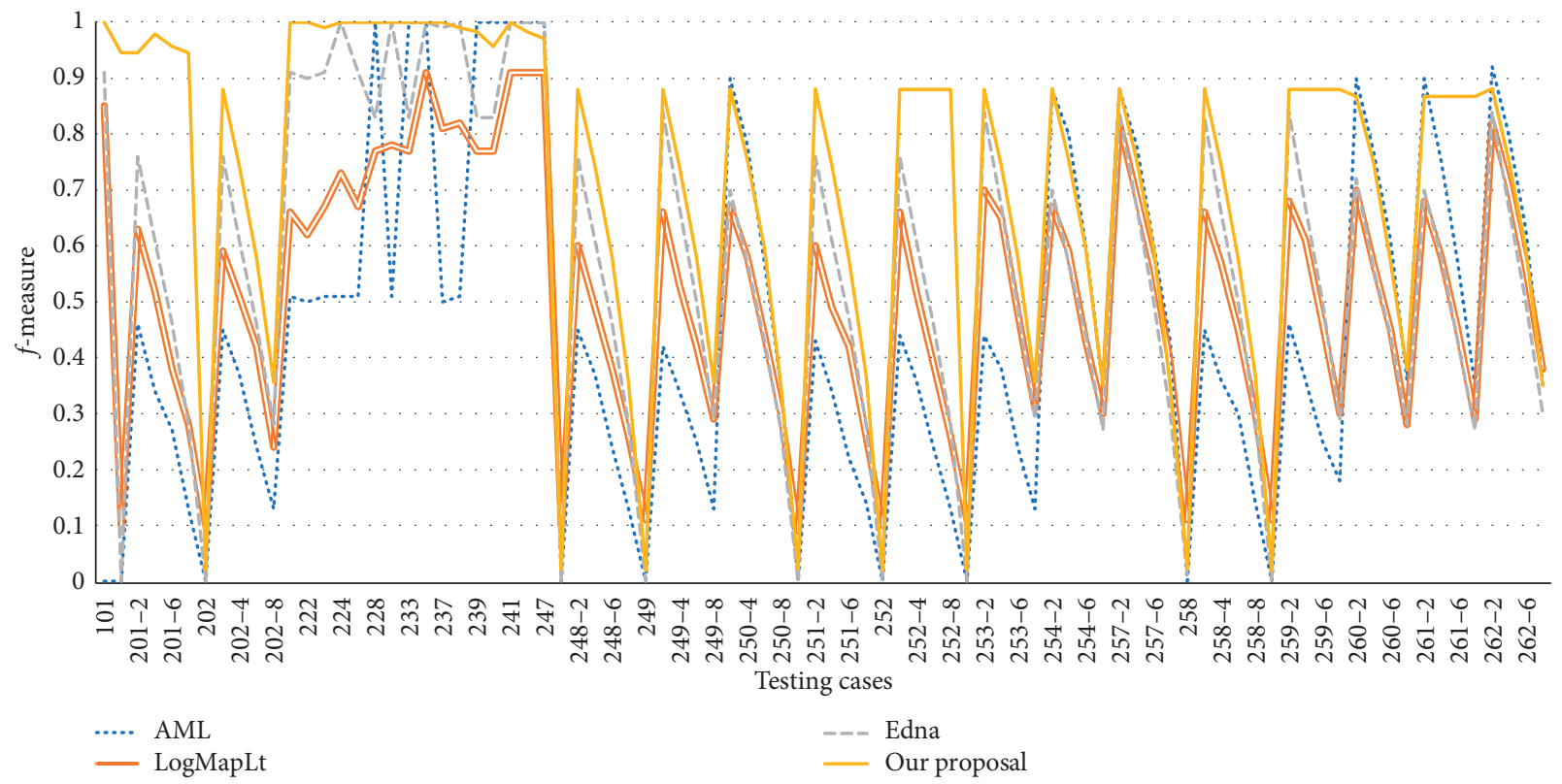

Figure 5: Comparison with OAEI's participants in terms of $f$-measure.

TABLE 4: Comparison with OAEI's participants in terms of $f$-measure.

\begin{tabular}{lcccc}
\hline Testing case & AML & Edna & LogMapLt & Our proposal \\
\hline $202-8$ & 0.13 & 0.24 & 0.28 & 0.3553 \\
$248-8$ & 0.13 & 0.26 & 0.28 & 0.3553 \\
$249-8$ & 0.13 & 0.29 & 0.29 & 0.3553 \\
$251-8$ & 0.14 & 0.26 & 0.49 & 0.3687 \\
$253-6$ & 0.24 & 0.48 & 0.29 & 0.5794 \\
$253-8$ & 0.13 & 0.31 & 0.29 & 0.3553 \\
$258-8$ & 0.14 & 0.3 & 0.28 & 0.3687 \\
$260-8$ & 0.36 & 0.28 & 0.3779 \\
\hline
\end{tabular}

are mentioned above, i.e., Levenshtein-similarity, Jaro similarity metric, WordNet-based distances, and similarity flooding in terms of recall, precision, and $f$ - measure.

Figure 6 depicts the result of our scheme for matching six pairs of real sensor ontologies and compares them with four basic EMMs, and the results show our proposal typically achieves very high capacity with the golden alignment. Furthermore, the application of fuzzy measure extends the single-dimensional evaluation on similarities judging by basic similarity measures to three-dimensional assessment, which fully express the similarity to gain a high-quality alignment.

In a word, FDM can significantly improve the accuracy of search results, and at the same time ensure a high recall rate, in all kinds of matching tasks that are superior to other competitors. 


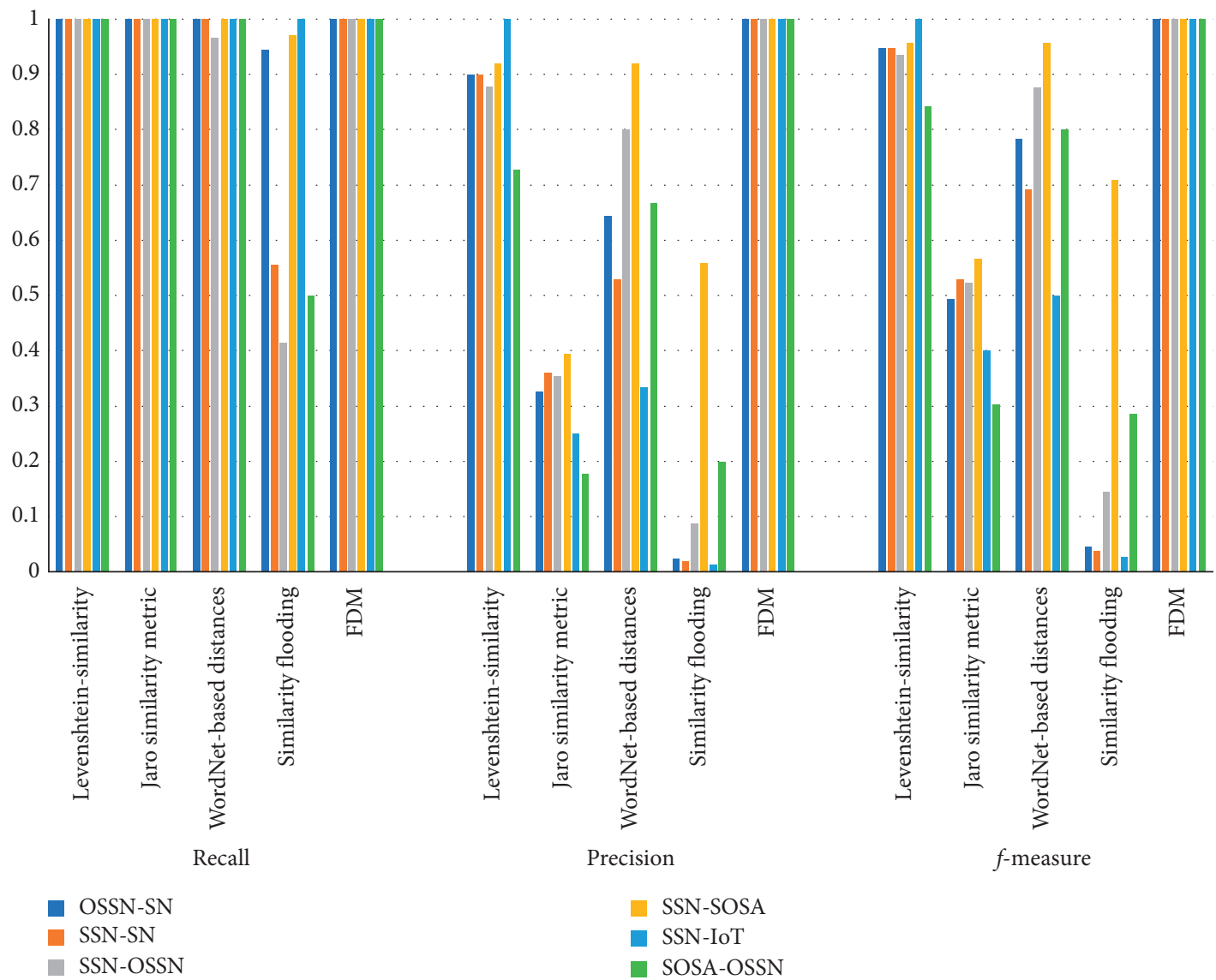

FIgURE 6: Comparison on two pairs of real sensor ontologies with four matchers.

\section{Conclusions}

Semantic connections among different sensor ontologies are of great significance to Nx-IIoT's communication quality and information security [50]. Therefore, a sensor ontology aggregating method based on Fuzzy Debate Mechanism is proposed, which extracts the ultimate alignment by performing arguments between different entity matching measures. A fuzzy similarity measure is presented to improve the alignment's quality, which models two entities' similarity in the vector space and their semantic distance is calculated by using cosine function. The Bibliographic tracks provided by OAEI and five real sensor ontologies were used to calculate the performance of the proposed method in this experiment. Compared with the most advanced ontology matching technology and four basic ontology matchers, the robustness and effectiveness of our proposal are verified.

Looking to the future, there are two challenges in sensor ontology alignment extraction technique: one is to measure entity similarity, and the other is how to develop extraction rules to tune the quality of alignment. In one hand, we need to further innovate the similarity measurement of domainspecific ontology to adapt to its fine-grained and complex structure. In the other hand, there is the need of approaches that can deal with the problem of uncertainty generated in the matching process. [50].

\section{Data Availability}

The data used to support the findings of this study are available from the corresponding author upon request.

\section{Conflicts of Interest}

The authors declare that they have no conflicts of interest in the work.

\section{Acknowledgments}

This work was supported by the Natural Science Foundation of Fujian Province (No. 2020J01875) and the National Natural Science Foundation of China (Nos. 61773415, 61801527, and 61103143).

\section{References}

[1] H. Liu, Y. Wang, and N. Fan, "A hybrid deep grouping algorithm for large scale global optimization," IEEE Transactions on Evolutionary Computation, vol. 24, no. 6, pp. 1112-1124, 2020.

[2] J. Pan, P. Song, S. Chu et al., "Improved compact cuckoo search algorithm applied to location of drone logistics hub," Mathematics, vol. 8, no. 3, pp. 1-19, 2020.

[3] J. Xiong, H. Liu, B. Jin et al., "A lightweight privacy protection scheme based on user preference in mobile crowdsensing," 
Transactions on Emerging Telecommunications Technologies, vol. 23, no. 6, pp. 1-16, 2020.

[4] J. Xiong, M. Zhao, M. Bhuiyan et al., "An AI-enabled threeparty game framework for guaranteed data privacy in mobile edge crowdsensing of IoT," IEEE Transactions on Industrial Informatics, vol. 17, no. 2, pp. 922-933, 2021.

[5] J. Lin, G. Srivastava, Y. Zhang et al., "Privacy preserving multiobjective sanitization model in 6G IoT environments," IEEE Internet of Things Journal, vol. 8, pp. 5340-5349, 2020.

[6] D. Bunker, L. Levine, and C. Woody, "Repertoires of collaboration for common operating pictures of disasters and extreme events," Information Systems Frontiers, vol. 17, no. 1, pp. 51-65, 2015.

[7] T. Wu, T. Wang, Y. Lee et al., "Improved authenticated key agreement scheme for fog-driven IoT healthcare system," Security and Communication Networks, vol. 2021, Article ID 6658041, 16 pages, 2021.

[8] F. Alamdar, M. Kalantari, and A. Rajabifard, "Towards multiagency sensor information integration for disaster management," Computers, Environment and Urban Systems, vol. 56, pp. $68-85,2016$.

[9] E. U. Ogbodo, D. Dorrell, and A. M. Abu-Mahfouz, "Cognitive radio based sensor network in smart grid: architectures, applications and communication technologies," IEEE Access, vol. 5, pp. 19084-19098, 2017.

[10] C. Michael, B. Payam, B. Luis et al., "The ssn ontology of the w3c semantic sensor network incubator group," Web Semantics: Science, Services and Agents on the World Wide Web, vol. 17, pp. 25-32, 2012.

[11] M. Ganzha, M. Paprzycki, W. Pawłowski et al., "Semantic interoperability in the Internet of Things: an overview from the INTER-IoT perspective," Journal of Network and Computer Applications, vol. 81, pp. 111-124, 2017.

[12] K. Janowicz, A. Haller, S. J. D. Cox et al., "SOSA: a lightweight ontology for sensors, observations, samples, and actuators," Journal of Web Semantics, vol. 56, pp. 1-10, 2019.

[13] P. Barnaghi, W. Wang, C. Henson et al., "Semantics for the Internet of Things: early progress and back to the future," International Journal on Semantic Web and Information Systems, vol. 8, no. 1, pp. 1-21, 2012.

[14] X. Xue, C. Yang, C. Jiang et al., "Optimizing ontology alignment through linkage learning on entity correspondences," Complexity, vol. 2021, Article ID 5574732, 12 pages, 2021.

[15] X. Xue, H. Yang, J. Zhang et al., "An automatic biomedical ontology meta-matching technique," Journal of Network Intelligence, vol. 4, no. 3, pp. 109-113, 2019.

[16] N. Alboukaey and A. Joukhadar, "Ontology matching as regression problem," Journal of Digital Information Management, vol. 16, no. 1, pp. 34-42, 2018.

[17] M. A. Khoudja, M. Fareh, and H. Bouarfa, "Ontology matching using neural networks: survey and analysis," in Proceedings of the 2018 International Conference on Applied Smart Systems (ICASS), pp. 1-6, Medea, Algeria, November, 2018.

[18] M. T. Dhouib, C. F. Zucker, and A. G. B. Tettamanzi, "An ontology alignment approach combining word embedding and the radius measure," in Proceedings of the International Conference on Semantic Systems, pp. 191-197, Karlsruhe, Germany, September, 2019.

[19] A. Ali, M. Hamid, K. Ahmad et al., "Context aware instance matching through graph embedding in lexical semantic space," Knowledge-Based Systems, vol. 186, pp. 422-433, 2019.
[20] F. Ali, K. Kwak, and Y. Kim, "Opinion mining based on fuzzy domain ontology and support vector machine: a proposal to automate online review classification," Applied Soft Computing, vol. 47, pp. 235-250, 2016.

[21] A. Siham, M. Sihem, and F. Muhammad, "Decision trees in automatic ontology matching," International Journal of Metadata, Semantics and Ontologies, vol. 11, no. 3, pp. 180190, 2016.

[22] G. Acampora, P. Avella, V. Loia et al., "Improving ontology alignment through memtic algorithms," in Proceedings of the 2011 IEEE International Conference on Fuzzy Systems (FUZZIEEE 2011), pp. 240-259, Taipei, Taiwan, June, 2010.

[23] M. G. Jorge, A. Enrique, and F. A. M. José, "Optimizing ontology alignments by using genetic algorithms," in Proceedings of the workshop on nature based reasoning for the semantic Web, pp. 1-15, Karlsruhe, Germany, October, 2008.

[24] M. G. Jorge and F. A. M. Jose, "Evaluation of two heuristic approaches to solve the ontology meta-matching problem," Knowledge and Information Systems, vol. 26, no. 2, pp. 225247, 2011.

[25] A. L. Ginsca and I. Adrian, "Using a genetic algorithm for optimizing the similarity aggregation step in the process of ontology alignment," in Proceedings of the 9th RoEduNet IEEE International Conference, pp. 118-122, Sibiu, Romania, June, 2010.

[26] Z. Xu, W. Zhang, T. Zhang et al., "HRCNet: high-resolution context extraction network for semantic segmentation of remote sensing images," Remote Sensing, vol. 13, no. 71, pp. 1-23, 2021.

[27] X. Xue and J. Chen, "Optimizing sensor ontology alignment through Compact co-firefly algorithm," Sensors, vol. 20, no. 7, pp. $1-15,2020$.

[28] X. Xue and J. Zhang, "Matching large-scale biomedical ontologies with central concept based partitioning algorithm and adaptive Compact evolutionary algorithm," Applied Soft Computing, vol. 106, p. 107343, 2021.

[29] X. Xue, X. Wu, C. Jiang et al., "Integrating sensor ontologies with global and local alignment extractions," Wireless Communications and Mobile Computing, vol. 2021, pp. 1-10, Article ID 6625184, 2021.

[30] V. Franzoni and A. Milani, "Semantic context extraction from collaborative networks," in Proceedings of the 2015 IEEE 19th International Conference on Computer Supported Cooperative Work in Design (CSCWD), pp. 131-136, Calabria, Italy, May, 2015.

[31] P. Buitelaar and M. Sintek, "Ontolt version 1.0: middleware for ontology extraction from text," in Proceedings of the $3 \mathrm{rd}$ International Semantic Web Conference (ISWC), pp. 1-4, Hiroshima, Japan, November 2004.

[32] M. Gaeta, F. Orciuoli, S. Paolozzi et al., "Ontology extraction for knowledge reuse: the e-learning perspective," IEEE Transactions on Systems, Man, and Cybernetics-Part A: Systems and Humans, vol. 41, no. 4, pp. 798-809, 2011.

[33] L. Laera, V. Tamma, J. Euzenat et al., "Reaching agreement over ontology alignments," in Proceedings of the International Semantic Web Conference, pp. 371-384, Athens, GA, USA, November 2006.

[34] L. Laera, I. Blacoe, V. Tamma et al., "Argumentation over ontology correspondences in mas," in Proceedings of the 6th international joint conference on Autonomous agents and multiagent systems, pp. 1-8, Honolulu, HI, USA, May, 2007.

[35] C. T. dos Santos and J. Euzenat, "Consistency-driven argumentation for alignment agreement," in Proceedings of the 5th 
ISWC workshop on ontology matching (OM), pp. 37-48, Shanghai, China, November 2010.

[36] S. Fernandez, I. Marsa-Maestre, J. R. Velasco et al., "Ontology alignment architecture for semantic sensor web integration," Sensors, vol. 13, no. 9, pp. 12581-12604, 2013.

[37] K. Todorov, P. Geibel, and C. Hudelot, "A framework for a fuzzy matching between multiple domain ontologies," in Proceedings of the International Conference on KnowledgeBased and Intelligent Information and Engineering Systems, pp. 538-547, Kaiserslautern, Germany, September, 2011.

[38] K. Todorov, C. Hudelot, A. Popescu et al., "Fuzzy ontology alignment using background knowledge," International Journal of Uncertainty, Fuzziness and Knowledge-Based Systems, vol. 22, no. 1, pp. 75-112, 2014.

[39] Y. Zhang, A. Panangadan, and V. K. Prasanna, "UFOM: unified fuzzy ontology matching," in Proceedings of the 2014 IEEE 15th International Conference on Information Reuse and Integration (IEEE IRI 2014), pp. 787-794, Redwood City, CA, USA, August 2014.

[40] V. Cross, "Fuzzy semantic distance measures between ontological concepts," in Proceedings of the IEEE Annual Meeting of the Fuzzy Information, vol. 2, pp. 635-640, Banff, $\mathrm{AB}$, Canada, June 2004.

[41] H. Neuhaus and M. Compton, "The semantic sensor network ontology," in Proceedings of the 12th AGILE International Conference on Geographic Information Science: Pre-Conference Workshop Challenges in Geospatial Data Harmonisation, pp. 1-33, Hannover, Germany, June 2009.

[42] K. Taylor, A. Haller, M. Lefrançois et al., "The semantic sensor network ontology, revamped," in Proceedings of the 18th International Semantic Web Conference, New Zealand, October 2019.

[43] M. Diaz, C. Martín, and B. Rubio, "State-of-the-art, challenges, and open issues in the integration of Internet of things and cloud computing," Journal of Network and Computer Applications, vol. 67, pp. 99-117, 2016.

[44] R. Ronald and Yager, "Uncertainty modeling using fuzzy measures," Knowledge-Based Systems, vol. 92, pp. 1-8, 2016.

[45] J. Euzenat and P. Shvaiko, Ontology Matching, Springer, Heidelberg, Germany, 2007.

[46] W. W. Cohen, P. Ravikumar, and S. E. Fienberg, "A comparison of string distance metrics for name-matching tasks," in Proceedings of the 2003 International Conference on Information Integration on the Web (IIWeb), pp. 73-78, Acapulco, Mexico, August 2003.

[47] G. A Miller, "WordNet: a lexical database for English," Communications of the ACM, vol. 38, no. 11, pp. 39-41, 1995.

[48] S. Melnik, H. Garcia-Molina, and E. Rahm, "Similarity flooding: a versatile graph matching algorithm and its application to schema matching," in Proceedings of 18th International Conference on Data Engineering, pp. 117-128, San Jose, CA, USA, March 2002.

[49] E. Jerome, "Semantic precision and recall for ontology alignment evaluation," in Proceedinds of the International Joint Conferences on Artificial Intelligence, vol. 7, pp. 348-353, Hyderabad, India, January 2007.

[50] T. Wu, L. Yang, Z. Lee et al., "Improved ECC-based threefactor multiserver authentication scheme t," Security and Communication Networks, vol. 2021, pp. 1-14, Article ID 6627956, 2021. 